\title{
An Overview of Lessons Learned from Sonic-boom Flight Research Projects Conducted by NASA Armstrong Flight Research Center
}

\author{
Erin. R. Waggoner, ${ }^{1}$ Larry J. Cliatt $\mathrm{II}^{2}$ \\ NASA Armstrong Flight Research Center, Edwards, California, 93523, USA \\ Michael A. Hill ${ }^{3}$ \\ Jacobs Technology, Fort Walton Beach, Florida, 32547, USA \\ and \\ Edward A. Haering Jr. ${ }^{4}$ \\ NASA Armstrong Flight Research Center, Edwards, California, 93523, USA
}

\begin{abstract}
Over the course of four years, a team of aerospace engineers at the National Aeronautics and Space Administration Armstrong Flight Research Center (Edwards, California) completed four projects, each with the objective to research sonic-boom signatures from a ground- and building-level perspective. The relatively compressed timeline of these projects resulted in many lessons learned. With each successive project, these lessons have been more relied upon and referenced. This paper provides a high-level overview of the relevant lessons learned and the importance of these lessons to future projects.
\end{abstract}

\section{Nomenclature}

$\begin{array}{ll}\text { AFRC } & =\text { Armstrong Flight Research Center } \\ \text { ATC } & =\text { air traffic control } \\ \text { B\&K } & =\text { Brüel \& Kjær } \\ \text { EAFB } & =\text { Edwards Air Force Base } \\ \text { EMI } & =\text { electromagnetic interference } \\ \text { FaINT } & =\text { Farfield Investigation of No-boom Thresholds } \\ \text { GPS } & =\text { global positioning system } \\ \text { LMR } & =\text { land mobile radio } \\ \text { NASA } & =\text { National Aeronautics and Space Administration } \\ \text { PC } & =\text { personal computer } \\ \text { PI } & =\text { Principle Investigator } \\ \text { POC } & =\text { point of contact } \\ \text { SCAMP } & =\text { Superboom Caustic Analysis and Measurement Project } \\ \text { SonicBOBS } & =\text { Sonic Booms on Big Structures } \\ \text { WSPR } & =\text { Waveforms and Sonic boom Perception and Response }\end{array}$

\section{Introduction}

The steady progression of sonic-boom flight research projects over a four-year period by the core sonic-boom team at the National Aeronautics and Space Administration (NASA) Armstrong Flight Research Center (AFRC) (Edwards Air Force Base, California) resulted in many lessons learned. For the purposes of this paper, sonic-boom tests are

\footnotetext{
${ }^{1}$ Aerospace Engineer, Research Aerodynamics and Propulsion Branch, AIAA nonmember.

${ }^{2}$ Aerospace Engineer, Research Aerodynamics and Propulsion Branch, AIAA nonmember.

${ }^{3}$ Aerospace Engineer, Research Aerodynamics and Propulsion Branch, AIAA nonmember.

${ }^{4}$ Aerospace Engineer, Research Aerodynamics and Propulsion Branch, AIAA nonmember.
} 
defined as "tests conducted for the purpose of researching sonic-boom signatures on a ground and building level." The lessons learned span the entire typical project life cycle, beginning with the planning phase and ending with the data analysis and reporting phases. An overview of the most pertinent lessons learned from the four projects is presented, including lessons concerning the pre-flight phase, methods of data collection, the flight phase, mishaps and close calls, and the post-flight phase.

The projects referenced within this paper include Sonic Booms on Big Structures (SonicBOBS) [Ref. 1], the Superboom Caustic Analysis and Measurement Project (SCAMP) [Ref. 2], Waveforms and Sonic boom Perception and Response (WSPR) [Ref. 3], and Farfield Investigation of No-boom Thresholds (FaINT) [Refs. 4, 5]. All of these projects were funded by the Commercial Supersonic Technology (CST) project within the NASA Aeronautics Research Mission Directorate.

\section{Pre-flight Phase}

Detailed attention within the formulation phase on any project is critical to increasing the likelihood of success. During project formulation, project concepts and technologies are identified and developed, the structure of the team is determined, and the preliminary design is completed. The formulation phase within each project helps to establish the groundwork and scope of a project and defines the project in sufficient detail to form an initial baseline capable of meeting mission needs.

Sonic-boom flight research formulation is anchored on four primary components: project planning, test deployment planning, project coordination, and logistics and test scheduling.

\section{A. Project Planning}

The key components of project planning focus on defining clear objectives and requirements. Project planning also includes selecting the test site and performing extensive collaboration with inter-agency and industry partners.

\section{Definition of Test Objectives and Requirements}

An Objectives and Requirements Document (ORD) should be the basis for determining and documenting all sonicboom research project requirements. The ORD defines the goals of the project, what is needed to achieve the defined goals, and how the project can show that the goals were met. While there is a well-documented process for defining NASA project requirements, particular lessons were learned concerning the application of this process to sonic-boom research.

It is critical to understand and properly define project requirements early in the planning phase. In defining requirements the team must take great care to parse out "highly desired" objectives or capabilities from required objectives or capabilities. One method of accomplishing this task is to define what, at a minimum, must be accomplished to make the project "fully successful" and what additional accomplishments would be considered "stretch" objectives. Finally, in defining "stretch" objectives, the team should consider what might be critical to the success of future projects.

Project personnel should not only establish requirements early, but avoid "scope creep" by closely critiquing all revisions and additions to the existing requirements. Such a critique is important for all requirements, but is particularly important for airplane test-point requirements. Sonic-boom research requires a comprehensive airplane waypoint planning process, typically utilizing computer code written specifically for the project [Ref. 6]. If an additional test-point requirement is added after the waypoint planning procedure has been designed, it is both time- and resource-intensive to make the necessary changes to the computer code and procedures.

Previous sonic-boom flight research has shown that setting a realistic schedule early in the planning phase is a requirement, especially when partners are involved. While a vast majority of aerospace projects identify a test date with the understanding that delays could push the date back, this is less acceptable with a project that involves partners or test-site coordination or both; such is the case for sonic-boom-research-focused projects. A requirement must be made to set a test date as early as possible in the planning phase of the project. While project personnel may be wary of date-driven deadlines, setting a test date facilitates making arrangements with partners and coordinating assets, and thus is paramount to the successful outcome of the project.

\section{Selection of the Test Site}

The emphasis of sonic-boom flight research projects is not limited to airplane and flight operations, because nominally the objective is ground-operation-centric; thus, a large portion of project planning is choosing a test site. In general, test sites shall have the airspace restrictions that allow the required airplane maneuvers (such as supersonic flight over land) while providing adequate terrain and area on the ground to deploy any required instrumentation. Test 
sites shall also to be accessible to the project team and located such that acoustic disturbances are minimized. These requirements often translate to the test site being in a remote location.

Some lessons learned for the pre-flight phase test-site selection process for sonic-boom flight research include the importance of visiting each prospective test location so as to identify potential sources of extra noise and determine how to mitigate such noise. Ideally, a test site would be completely silent; however, no such site exists. "The quieter the better" is the simple rule of thumb. After visiting a potential test site it is imperative to discuss with the site owner the possibility of reducing extraneous noise. Past interactions taught the project team that people are often willing to make such accommodations.

Other topics to consider when selecting a test site include the presence and use of irrigation systems, and restrictions concerning equipment installation. For example, at outdoor test sites, project-required equipment is often held in place using stakes, but some landowners and jurisdictions require permits for stakes of a certain length.

Similar concerns apply to test sites involving structural response data. Additional considerations include determining what doors and windows can be propped open to allow instrumentation cable routing, checking to see if loud appliances can be turned off before testing, and making sure there are accessible electrical outlets.

Accessibility to the site itself is also of importance. When selecting a test site, the project team should become aware of any potential obstacles or hazards that might be encountered while traveling to the site. The FaINT project, in addition to the primary test site, had six potential remote test sites, called sentinel locations, which would each accommodate single microphones. Two of the remote sites proved to be too difficult to access because there was a high chance of a vehicle getting stuck in deep sand. The project team should make sure that there are multiple safe and accessible routes to and from the test site, and consider the implications of transporting heavy equipment to the site.

As there are many concerns when selecting a test site, project teams should be advised that site visits are critical to mission success and that one site visit is not sufficient. When selecting a test site, project teams should visit prospective test sites at least twice, preferably during the predicted testing hours; upon site selection, more visits should be conducted to determine whether any pertinent changes have occurred. Ultimately, the lessons learned regarding test-site selection can be summed up as follows: (1) selection of the test site must be meticulously thought out; and (2) multiple visits to the test site will reduce the number of acoustical and logistical unknowns.

\section{Collaborating with Partners}

The NASA sonic-boom flight research projects typically involve a number of partners. Partnering with outside organizations and communities is facilitated and governed by Space Act Agreements (SAAs). While SAAs allow NASA to enter into partnerships with organizations that give NASA access to a wider range of technologies and capabilities that are not part of NASA core competencies, it does take time to set up these legal agreements. Early sonic-boom flight research projects, predating SonicBOBS, created partnerships through invited contributions, in which partners are involved in projects through voluntary participation. In these instances any assets from partners cannot be mission-critical or a requirement and are the responsibility of the owner. Note that if data are to be exchanged as a part of the agreement, then a formal agreement (such as a Space Act Agreement) must be established as early as possible to ensure that no misunderstandings result in schedule delays to the project.

Because partnership is usually entered into on a voluntary basis, there are no contractual deadlines for the partners to meet, which can result in difficulty identifying which partners will be participating, and what assets (both in terms of workforce and equipment) each will be providing. Future project teams should implement a hard deadline by which potential partners should decide and communicate whether they will be participating, and a similar deadline should be imposed for identifying what assets each partner will contribute; both of these deadlines should be determined early in the planning phase, noted in project documentation, and communicated to prospective partners.

It is important to define the expectations of each organization participating in the test. Setting such expectations is especially important regarding partners of whose influence and authority is a requirement. For example, during the WSPR project, a two-week delay was experienced due to a lack of documentation outlining each stakeholder's understanding and expectations of the project. Similar future tests should establish approval and agreements between partners early in the project, document all stakeholder expectations, and communicate such expectations to all remaining stakeholders.

For sonic-boom human-response research, a critical partnership is with the human-response subjects themselves. Public participation as test subjects is a requirement, so an established partnership is vital. There are two lessons learned from the WSPR project regarding partnership with human subjects: (1) recruitment of volunteers requires a significant amount of time, effort, and incentive; and (2) continued contribution throughout the test by the volunteers is not guaranteed and providing the incentive at the end of the test mitigates volunteer dropout. 


\section{B. Test Deployment Planning}

Sonic-boom flight research nominally emphasizes personnel, instrumentation, and equipment on the ground. The ground assets are required to measure the sonic booms produced by the airplane, and are therefore vital to the success of the project. Unique requirements concerning ground assets often result in project teams being deployed to a remote test site. For NASA sonic-boom flight research projects a remote deployment is generally considered to be any ground-test site outside of Edwards Air Force Base (EAFB). A test located on EAFB but not near NASA AFRC is considered a local deployment due to additional complexities concerning the transportation of personnel and equipment, as well as the need to coordinate basic amenities for the field crew. Concerning any type of deployment, some of the aspects that have garnered the most lessons learned involve understanding the reasons for test deployment, the financial impact of test deployment, and the complexity a deployment adds to a project.

\section{Reasons for Test Deployment}

Some of the rationale for utilizing a deployed test location for a sonic-boom flight research project include avoiding local noise sources, having enough area to fit the desired instrumentation arrays, avoiding placing sonic booms on populated regions, or requiring particular climatological or geographical features.

The SCAMP was one of the few sonic-boom flight research projects in the past 20 years to be remotely deployed (near Cuddeback Dry Lake, California) that was originally planned to be locally deployed. Early analysis, however, revealed that SCAMP would likely produce exceptionally loud sonic booms (levels above $12 \mathrm{psf}$ ) over a large portion of the sonic-boom footprint. Sonic booms at these noise levels can be harmful to human hearing; the sonic-boom carpet planned for the SCAMP was too large and unpredictable to consistently keep away from populated areas, thus the remote deployment.

In this case, the site selection process resulted in the lesson learned that it is equally as important to understand why proposed test site location cannot be selected as it is to understand why a test site can be selected. Such an understanding helps clarify to all stakeholders why the project must work with the added cost and complexity of remote deployment if the project is to be successful.

\section{Cost Impact and Added Project Complexity}

The deployment of a project has a significant cost impact on the project. For the SCAMP, the major additional costs included truck operations to transport equipment, personnel lodging, meals and incidental expenses, and 24-hour security guards. Security was required because the SCAMP was located on a publicly accessible site over 11 miles from the nearest well-traveled road, causing concern for equipment integrity. These major expenses accounted for a significant percentage of the total flight operations budget.

A remote deployment also adds other considerations, which can significantly increase the amount of planning and coordination required, that would not be necessary for a test site located on EAFB, near NASA AFRC. The project team must plan how to get equipment to the test site, which may not be a trivial task. In addition to ground equipment, a remote deployment might be located such that it is not possible to use the Dryden Aeronautical Test Range for flight operations, or making it impractical to use AFRC aircraft maintenance facilities. In such cases, these capabilities would need to be identified and acquired at the test deployment site.

Other complexities of test deployment might include the need for amenities such as at least two portable toilets, plenty of drinking water for the field crew, automobile jumper cables, shelter and eating areas for the crew, a way to charge batteries, et cetera. Crew rest is also critical and needs to be accounted for. Crew rest is complicated by the long travel time usually associated with test deployment sites and by the fact that equipment batteries usually need to be charged in another location, lengthening the duty day.

The lesson learned is that the complexity of basic logistics - transporting equipment and essential amenities, for example - dramatically increases as a function of the remoteness of the test site and whether or not access to the test site is limited. A remote or publicly accessible test site requires the project team to consider all logistics with greater scrutiny.

\section{Project Coordination}

The effort involved in coordinating a large test makes it necessary to assign each project team member a domain of coordination tasks to spearhead. A key lesson learned is that it is critical that the project team take time to sit down and work out a plan of action for each part of the test - from the receipt and set-up of test equipment to teardown and equipment return shipping. Taking the time to work out such a plan allows the project team to present a unified front throughout each phase of the test and greatly reduces the likelihood that a task will be overlooked. Such a discussion also eliminates confusion as to who is the point of contact (POC) for various coordination tasks, thus minimizing potential confusion with coordinating organizations. Furthermore, working through the process of creating a plan of 
action forces the project team to look at the big picture of the project, which is essential to identifying any requirements for additional workforce or the need for skill sets not found within the core team of the project. Once the need for resources external to the project team has been identified, it is critical to communicate these needs and coordinate the additional equipment and staffing as soon as possible.

During the coordination effort, the project team must begin interacting with the aircrew, with added emphasis on discussions regarding flight profiles. Coordination with the aircrew is crucial during project planning, especially for a uniform understanding of the mission profile and daily schedule; such interaction and subsequent buy-in from the aircrew will present a united front to management during practice flights, technical briefs, and other project reviews.

\section{Coordinating with Government Entities}

All of the aforementioned tests, as noted above, with the exception of the SCAMP, occurred on EAFB, but many of the resultant lessons learned are relevant to coordination with other military bases and government agencies.

In working with external organizations, one of the biggest lessons learned is that reducing the required direct oversight said external organizations during the test window is highly desirable. Mitigation of these logistics requires extra effort in the coordination phase but significantly eases the workload on the field crew during the test phase.

There are five general groups that will need to be involved in the coordination and execution of most tests that involve government assets. These organizations are: Environmental, Air Traffic Control, Airfield Management, Frequency Management, and Security (Access to Military Bases, and Facilities Use). These specific titles cover general topics that must be addressed prior to any test; the organization names might differ across test sites.

Environmental - Site selection of primary and backup test locations should be completed as early as possible, as an environmental survey is required prior to the use of any government land for testing purposes. This survey often requires considerable time due to the heavy workload of these environmental organizations, the paperwork required to start the process, and the implications that it may place on the test.

Another reason to complete the environmental survey as soon as possible is to accommodate any concerns discovered during the survey process, such as wildlife or undetonated munitions precautions. Frequently, wildlife-related training for the field crew is required, and tests on a remotely-located section of an active or inactive military base should warrant awareness of how to react to the discovery of undetonated munitions. This awareness includes communicating the possibility of finding undetonated munitions to all field personnel, having a plan of what to do in the event that a suspect object is encountered, and briefing this plan to all crewmembers prior to deployment.

The key lesson learned regarding coordination with any environmental department is that it is critical to get the environmental survey performed as early as possible, as the results of the survey could delay the project schedule by requiring a change to equipment set-up procedures, crew training requirements, or the test site location itself.

Air Traffic Control - If instrumentation will be in an air traffic controlled (ATC) limited-access area, the project team must meet with ATC personnel during the project coordination phase. It is crucial to discuss and develop standard operating procedures concerning access, entry and exit, emergency procedures, nominal communications, and the deployment of any unusual equipment early in the pre-flight planning phase of the project.

The sonic-boom teams had positive interactions with Edwards ATC concerning ground operations and the operation of unusual equipment during the FaINT project test window, due largely to the pre-coordination and communication with ATC personnel prior to test execution. The lesson learned is that establishing detailed communication with ATC early in the pre-flight planning phase will increase the likelihood that day-to-day operations run smoothly throughout the flight phase.

Another consideration concerning interactions with ATC is planning the flight path of the test airplane(s). While airspace constraints on flight paths are addressed during mission planning, it is also important to consider what artificial effect instrumentation error has on the airplane trajectory that is displayed to ATC. For instance, in the past, known typical pitot-static calibration limitations resulted in false reports of the airplane climbing dramatically during transition through Mach 1.0, which caused ATC to become concerned and relay these concerns to the aircrew during critical moments of the test. The lesson learned for future projects is to communicate such problems to ATC before testing, and prepare for these possibilities in flight-path planning.

Airfield Management - If the project will require instrumentation or personnel to be placed anywhere on an airfield, airfield management must approve. While the need to contact governing and partnering organizations early in the planning and coordination of a project has been emphasized, airfield management is one of the most critical organizations with which to establish communication early in the life cycle of the project. Initial coordination with airfield management should begin at the same time as coordination with environmental has been initiated. 
Early interaction with airfield management is critical because this group is the scheduling authority for airfield assets and may place restrictions on the project team concerning the layout, set-up, and teardown of equipment. As well, airfield management has complete control over access to all airfield assets, meaning that the airfield can be shut down with little notice, particularly in the event of an emergency, precipitation, or severe weather. Additionally, if the lakebed has been closed due to rain, the closure can last for quite a long time - sometimes up to several months during the winter. As such, it is wise to avoid scheduling a test on the lakebed during a rainy season. Lessons learned are: (1) the project team needs to communicate all desires and expectations with airfield management up front, while being aware of all of the expectations airfield management has concerning the project and the general operation of the airfield; and (2) test-site selection can be weather-dependent - the time of year that the site will be used can impact project risk. Discussion with the organization in control of the site in question will give the project team the best idea of how weather-dependent the site is, and how much risk the project is assuming.

Frequency Management - The use of radio frequencies for transmission of data or communications is well regulated on military bases and government installations. Therefore, approval is necessary before operating any wireless sensors or equipment equipped with a "transmit" or "receive" function from the Frequency Management group of the installation prior to operation of such equipment. Future test teams should be aware that the process of gaining approval to use a wireless device on government property can be lengthy, and plan to submit the requisite documentation several months in advance of the test. Note that even if the test takes place off of a federal installation, if the organization leading the test is government-sponsored, then it is likely that all equipment capable of transmitting or receiving a signal will be required to go through a frequency management process.

Concerning field communications, discussed in greater detail below, it is also advised that future test teams be aware that very high frequency (VHF) and land mobile radios (LMRs) have already been approved for use on military bases, but a frequency request must be submitted prior to their use. Commercially available camping-style walkietalkies or family radios are not approved for use on military bases, nor are they approved for use by government personnel during a government-led test.

The lesson learned is that it is important to ensure that all equipment has been approved for use by the government well before it is used on government land. This approval is accomplished by discussing restrictions with a frequency management group and is best done before the test setup design is finalized.

Access to Military Bases / Government Facilities - Project personnel at NASA AFRC collaborate with several outside organizations to complete sonic-boom flight research projects. This collaboration is highly beneficial to gathering a larger, more specialized data set, but it also means that the logistics of getting off-base personnel access to the installation and physically onto the test site becomes a bigger, more time-consuming task. The key lessons learned concerning base access are: (1) designate a person to serve as the POC for base access with the understanding that they will essentially be on call for the duration of the test; and (2) the base access POC should not be a member of the field crew to avoid overtasking or crew rest issues; a NASA project manager is a good fit for the base-access POC role. Access for all visiting members of the test team who are United States citizens should be addressed simultaneously, roughly two months before the test, to allow for the time it takes to gather the information from the personnel, complete all the required forms, and ensure their access is granted by base security. The base access POC should contact base security to discuss current procedure for granting base access to a large group. Another lesson learned concerning base access is that the POC should plan to meet arriving visiting personnel at the base access gate when the visitors initially arrive, and accompany them when they renew their passes.

Facilities Use - As previously mentioned, some of the prior sonic-boom tests focused on or included building-response data, which present unique coordination challenges. Lessons learned regarding site selection have taught the sonic-boom team that selecting a building to instrument and gaining permission to do so early in the pre-flight planning phase is essential to project success. One unique hurdle to instrumenting a building is that if the building is occupied the test team might need to coordinate with the owners and/or tenants of the building. Usually this coordination involves requesting occupants do not open doors or windows or operate heavy machinery, or perform other tasks that could alter the data during the time of testing. Making these requests early in the coordination or site-selection process helps the project team eliminate test sites that are not ideal because of an increased possibility of erroneous test measurements. Additional consideration should be given to the security of the test hardware, including how many people have access to the building, and whether it is acceptable to leave test equipment in the building overnight. Finally, facilities owners usually want to approve any equipment installation; in this case it is best to know the exact size, amount, and installation technique of the equipment in question as early as possible. 


\section{Coordination of Land and Airspace}

While most of the sonic-boom flight research projects discussed in this paper took place on EAFB, it is reasonable to assume that similar tests conducted out in the public would require, at a minimum, the same level of coordination. In fact, it may take much more time than anticipated to determine which organizations need to be involved in the coordination effort. For example, in the planning of the SCAMP the team had to discover the governing authority for Cuddeback Lake, California. The discussions with the Bureau of Land Management, the California Department of Fish and Wildlife, and the United States Air Force to determine the who had the authority required as much time as did the planning efforts that followed. Approval from an environmental authority might still be required, depending on what organization has authority over the test site. As with all other test sites, storage of test equipment overnight or over weekends needs to be discussed early, as this might not be allowed. Tests in communities will be accessible to the public, and thus present a greater concern for the security of hardware. Similar to building-response tests, the organization in control of the test site will likely want details concerning how the test equipment will be installed and how much equipment will be used; as usual, it is best to provide as accurate an answer as possible as early as possible in the life cycle of the project in order to prevent confusion and misunderstanding leading to work-arounds during the set-up stage. Coordination tasks often take more time than initially assumed, thus project teams should begin the coordination early in the pre-flight phase.

\section{Coordination with Partners}

While exchanging the appropriate technical details with partners is critical to test success, it is also important to begin coordinating non-technical logistics while the test setup is being designed. Of particular interest is the number of people who will be traveling to the test site and how many of those people will be working in the field. This information will provide the team with information on additional staffing requirements from personnel outside of the sonic-boom team is needed. Furthermore, the number of field crewmembers will drive how much vehicle support is needed, which is especially critical if the test site is in a remote location or a controlled-access area. It is important to discuss vehicle support requirements with each partner prior to the test to ensure that the expectations of all involved parties are well understood. The lesson learned in this case is to create a detailed ground-operations plan that covers all of the daily operations and a detailed list of which personnel will be assigned to each task, and when those tasks will take place.

Working on a military base or other similarly-regulated site requires the project team to follow a specific set of standard operating procedures and regulations, which are often unfamiliar to external partners. In previous tests these expectations and rules were communicated by electronic mail or orally, but the most successful method of communication is to distribute one document outlining all of the base rules and expectations. Each participant is then required to read the document and return the back page of the document signed and dated to indicate their understanding of, and agreement to operate under, the documented terms. It is also recommended that a comprehensive electronic mail message with the general test schedule and other pertinent information be sent to the partner organizations two weeks prior to the test window.

Finally, it is important that each partner knows and understands what the daily schedule will be during the test window. During morning setup, many activities occur simultaneously. If access to the test site is controlled, ferrying vehicles and equipment can require a complicated series of logistics that takes longer than it would if everyone could merely drive their rental car out to the test site. The recommendation is that the test team explain the morning timeline to the entire field crew during the Day One briefing so that project team members understand how they fit into the bigger picture of the daily activities as well as the daily, detailed ground operations schedule.

\section{Navigating Potential Roadblocks}

Despite the number of sonic-boom ground measurement tests the NASA AFRC sonic-boom team have performed, and how well-versed the team is in coordinating such tests, there are a few commonly encountered roadblocks. Some of these roadblocks concern the perception of sonic booms and being involved in a test focused on them; being involved with an ongoing test; equipment integrity and security; and avoiding superfluous noises.

Sonic-boom-related Stigma - The term "sonic boom" carries a specific connotation to most people. Some people do not mind sonic booms; however, there is a segment of the population that has strong negative reactions to the term. These perceptions may be based on previous personal experiences and dislikes, or based on word-of-mouth anecdotes. Essentially, there exists a great deal of misinformation and negative perceptions surrounding sonic booms. The key lesson learned is that project teams planning future sonic-boom testing involving communities unfamiliar with sonic booms will need to educate the public prior to testing. Topics that should be addressed include sonic-boom carpets and how sonic-boom carpets can be strategically placed to reduce human exposure. 
When operating in an area where other organizations are flying supersonically it is common that all sonic booms produced during the publicized test window are attributed to the publicized project. This phenomenon is also associated with people familiar with flight-testing; EAFB personnel have asked NASA to rearrange flight schedules to de-conflict with large meetings. The lesson learned here is that tests involving the public need to be sensitive to community events in the creation and execution of the flight schedule.

When ground-measurement tests enter the realm of collecting human-response data, the situation becomes more complicated. Because of the history of human-response sonic-boom testing, the stigma attached to sonic booms, and the previous press resulting from sonic-boom tests, some within NASA expressed concern about whether NASA should perform or endorse this type of testing. The lesson learned is that the project team should clearly communicate the justification for human-response testing and test details with all project stakeholders, especially those with the authority to halt the project due to such concerns. This communication must begin in the earliest project formulation activities in order to ensure a successful project.

Use of Land and Facilities for Instrumentation - Sonic-boom stigma aside, it is possible that test-site selection could be challenging because some people are reluctant to participate in an ongoing test, particularly if requests are made of residents or occupants to avoid certain locations or activities during the test.

In the future, the desire to not be in close proximity to an ongoing test could present a challenge to sonic-boom response testing, especially if quantitative data would be gathered in community and residential areas to use as a reference against the qualitative response data. The concerns in this case would likely be the use of microphones to record sonic booms in neighborhoods and the perceived privacy implications; these concerns were in fact raised during the WSPR project. Utilizing a manual push button to record sonic booms, only using auto-triggers during flight windows, or obtaining approval from each neighborhood to continuously use auto-trigger settings are all potential ways to mitigate this problem in the future.

Equipment Integrity and Security - In an effort to reduce the workload on the field crew by decreasing daily setup and teardown requirements, some equipment is usually left set up for the duration of the test window. Often, cabling is left in place while microphones, recording hardware, and signal-processing hardware are picked up and stored near the recording site at the end of each day. This approach is optimal for a more reliable morning set-up and advantageous from the standpoint of crew rest, but presents problems concerning the integrity of the hardware by way of interference from people and wildlife.

Local wildlife present a constant problem to both the test team and the cabling. Rabbits and mice often chew on the cables overnight. Overnight storage, other wildlife concerns, and prevention of rodent chew-throughs are discussed below. Leaving equipment at the test site overnight also poses security problems. In controlled-access locations, storing equipment in watertight, durable cases that are then stacked near each recording location overnight has sufficed. During the WSPR project the equipment was secured using a locking cable or a locked chain looped around a telephone pole or other permanent fixture; none of the equipment thus secured was tampered with. During the SCAMP, the remote off-base test site was publicly accessible, and thus a night patrol of NASA security guards was added to the field crew. The lesson learned is to always consider the logistics of the entire day and to plan to secure all equipment against all threats: people, weather, mishandling, and wildlife.

\section{Logistics and Test Scheduling}

Planning the test schedule and detailed coordination of test operations for recent NASA sonic-boom flight research projects has provided many valuable lessons learned. Routine parts of sonic-boom flight research include setting the research flight phase schedule, planning the daily test schedules, and making pre-test preparations.

\section{Setting the Research Flight Phase Schedule}

Time is always a lacking commodity during the flight phase of a project, but the biggest lesson illustrated by previous sonic-boom flight research projects is that the project team must allow as much time for the flight phase as possible. Regardless of the reasons for this approach, it is highly suggested that more time be worked into the schedule to allow for data review between flights, pick up ground equipment, remove equipment from the test site, and give the crew adequate rest. Future teams should plan for this time in their schedule, which requires the team to be realistic about how much time each task will take and to set the expectations of all stakeholders.

A lesson learned from previous tests is that time should be made to look at all data between flights. When there are multiple flights in a day, the data should be looked at between each flight day. This process allows the project team to find problems with instrumentation systems or data collection methods, and provides the opportunity to plan future test points, which could include repeating test points based on data that have already been collected and viewed. 
A project team member could serve as a dedicated data reviewer, allowing the rest of the project team to focus on flight and ground operations.

Planned non-flight days within the flight phase are highly advised; the sonic-boom team will be implementing this strategy in future tests. The non-flight days could be dedicated to data review, but would also allow time to inspect equipment and make any necessary repairs. Non-flight days also provide the opportunity to re-train equipment operators if the data review shows that apparent data collection errors or other operational mistakes were noted. Nonflight days after the flight phase are suggested to better facilitate picking up any equipment. These lessons learned are discussed below.

\section{Planning the Daily Test Schedule}

Most NASA AFRC sonic-boom flight research projects have aggressive flight phases, often including multiple flights per day. The AFRC flight operations policies typically prohibit flights before dawn and after dusk due to the operational hours of the EAFB airfield. The ideal time of day for most sonic-boom research flights is early in the morning, when wind levels are low and the wind noise on any ground instrumentation thus is at a minimum. These limitations result in a relatively small window of time within which to execute multiple flights; therefore, a carefully planned and orchestrated daily schedule is necessary.

Radio Frequency Scheduling and Daily Meetings - Radio frequencies are required for communicating with airplane pilots and for telemetering any airplane data. The frequencies need to be scheduled and designated for each flight in order to prevent radio interference. Various circumstances (for example, weather delays or maintenance problems) can cause flight times to be volatile. Sonic-boom flight research projects have experienced extended delays and complications due to the need to reschedule airplane radio frequencies. The lesson learned is that project teams should schedule the necessary radio frequencies for the entire day when possible, if the project team intends to execute multiple flights over the course of a day. The sonic-boom teams have experienced fewer frequency-scheduling-related delays by employing this planning strategy.

An aggressive flight day can lend itself to confusion amongst personnel, especially when plans change or operative adjustments need to be made. Project teams must have mandatory morning meetings with the entire field crew each test day prior to morning setup. These meetings should be brief and provide an overview of the activities of the coming day. Daily morning meetings also enable updates or vital information to be communicated and can also offer an opportunity for crewmembers to share concerns or questions. This lesson was learned in some of the early sonic-boom flight research projects, and the daily morning meeting was then implemented during later tests. This change in operations resulted in less chaos and fewer delays during the daily equipment setups, resulting in an overall shorter duty day for the field crew.

Crew Rest - An aggressive flight day can also mean a very long workday for the ground crew. The planned daily test schedule for the SCAMP, which is a typical schedule for an aggressive sonic-boom flight research flight phase, is shown in table 1. 
Table 1. Planned daily schedule for the Superboom Caustic Analysis and Measurement Project (SCAMP).

- Setup and calibration of ground instrumentation

- Airplane instrumentation checks

(0400)

(0600)

- Pre-flight at beginning of flight week

- Day of flight each flight day

- Compute waypoints and deliver to pilots

- $\quad 1^{\text {st }}$ research flight takeoff

- Flight

- Field crew break

(0700)

- F-18 turn-around

- TG-14 turn-around

- $\quad 2^{\text {nd }}$ research flight takeoff

- Flight

- Field crew break

- F-18 turn-around

- TG-14 turn-around

- $\quad 3^{\text {rd }}$ research flight takeoff

- Flight

(1 hr)

(1 hr)

(2 hr)

$(2 \mathrm{hr})$

(1 hr)

(1 hr)

(2.5 hr)

(2 hr)

$(1 \mathrm{hr})$

Usually test days are 11.5-hour workdays (including two one-hour crew breaks). While an 11.5-hour day itself would be strenuous, crew breaks are typically spent working, and common flight operations problems often cause an extended test day. Thus, the resulting workday has often exceeded 14 hours. These extended days were not due to project expectations, but rather due to the high work ethic of the field crew. During a project with long days such as these, and a two-week flight campaign filled with back-to-back test days (as was the SCAMP), field-crew exhaustion became an alarming problem. The key lesson learned is that to avoid exhaustion, crew rest must be a priority when planning the daily flight schedule. This schedule must add more breaks and make them mandatory, include more field crew personnel so that tasks can be staggered, or simply make the flight schedule less aggressive.

Planning a less-aggressive flight schedule can be difficult when working with external partners. External partners often have set budgets that limit participation. Budgetary concerns have been one of the primary reasons for aggressive test schedules. As well, some other agencies and non-government companies have duty-day policies that differ significantly from those of NASA. In previous projects, these policy differences led partners to lobby for longer work days and many more airplane flights over the course of the flight campaign. The core lesson learned is that sonic-boom flight research projects must establish crew rest requirements early in the project-planning phase, and communicate these requirements to potential partners.

\section{Making Pre-test Preparations}

As with any flight research project there are a number of activities that take place after initial planning in preparation for the flight campaign. For NASA sonic-boom flight research projects, the typical pre-test preparations that garner the most lessons learned are inspecting the ground test site, receiving equipment from external partners, and the training of project personnel.

Test Site Checkout and Receiving Equipment - After selection of a test site, the entire - or at least the local - field crew needs to visit the test site prior to equipment setup. Such a trip assures familiarity, reduces surprises, and is especially beneficial to the personnel who will be delivering equipment to the test site. The site visit also allows the project team to figure out exactly how equipment will be mounted on buildings, if a structure is to be instrumented; stucco and tall buildings add a challenge. An early trip to the test site provides the opportunity to check the quality of radio communications as well as internet connectivity (mobile hotspots) and allows time for the project team to consider backup options. Test site reconnaissance is also used to generate high-fidelity maps that can be provided to 
the field crew. The routes (and backup routes) to the test site should be adequately marked; include reflectors if night access is anticipated. It is recommended that signs be distributed at least every $1,000 \mathrm{ft}$. along routes.

Partners often contribute to projects by offering technical expertise during planning and execution phases, and by providing field-crew personnel. Partner contributions are extremely valuable and fill a wide variety of project needs. The most common method of support is loaning ground instrumentation and equipment to NASA AFRC during the flight phase of a project. Thus, it is highly recommended that there be a staging and equipment storage area when expecting large quantities of equipment. The lesson for future projects is that even the smallest details in pre-test logistics make a vast difference in how the beginning of the test phase performs, and thus even small details in pre-test logistics deserve a methodical approach. Another lesson regarding loaned equipment is that projects should set a firm equipment delivery deadline. This deadline should allow for complications, including international customs delays. Without an equipment delivery deadline, project teams could risk not having time to perform a full system check before deployment.

Personnel Training - Another pre-test preparation that has yielded important lessons learned is personnel training, which includes location-specific training and equipment-operation training. Examples of previously encountered location-specific training courses include desert tortoise precautions, unexploded ordinance identification and precautions, and heat stress instructions. It is imperative that the project team work diligently to identify any training that will be required well in advance of field-crew deployment. Some of these training courses can be taken online, but for projects including many ground personnel, verifying that each person has taken the appropriate training can be tedious. The lesson for future projects is that project teams need to organize an initial team-training session after all ground personnel have reported for duty. If this session is not possible, training by way of videoconference or teleconference is a reasonable alternative.

In addition to location-specific training, there must be an introductory briefing before equipment is deployed. This briefing serves as an orientation and should include detailed information and documentation on how to deploy, set up, calibrate, and tear down all ground equipment. During the meeting any guidelines or location-specific rules need to be clearly stated. Guidance for sonic-boom recording etiquette should be provided. Specifically, NASA sonic-boom flight research requires that all ground personnel be silent for a predetermined amount of time prior to and after the sonic boom. Typically, silence should begin approximately two minutes before the estimated arrival of the sonic boom and end 15 seconds after the sonic boom has been heard. The meeting should also provide hands-on instruction for the use of radios and other communications devices. This instruction requires little time, but can immensely benefit the project.

During the introductory briefing, all field crew need to be educated on expected sonic-boom sounds, especially personnel who will be recording data or providing feedback during testing. An uneducated ear could result in poor feedback and, therefore, inaccurate airplane waypoints. Ideally, test-phase introductory briefings would include sample clips of the different sonic-boom classifications that are expected.

A similar briefing should be held for volunteer test subjects involved in sonic-boom human-response testing. The briefing should provide all volunteers with any information needed, including what is required of the volunteers, and any other training that might be necessary. The meeting should provide volunteers with training on how to properly submit their responses. All volunteers should receive the same information, thus, the number of times this briefing is held should be kept to a minimum.

\section{Methods of Data Collection}

The main thrust of each of the NASA sonic-boom flight research projects was to collect useful acoustic data to aid in the understanding of sonic booms. To collect these data, NASA and partners used a variety of methods to capture the acoustic and pressure signatures of the sonic boom at different locations and in different environments. There are two main types of arrays: the ground instrumentation, which captures the acoustic and pressure signatures of the booms at ground level; and the airborne instrumentation. While overall data collection was successful in the vast majority of runs for these tests, there were several lessons that were learned during the operation of these data acquisition systems throughout these campaigns.

\section{A. Ground Instrumentation}

The NASA AFRC uses a wide variety of ground instrumentation in each of its sonic-boom flight research tests. As a standard, this instrumentation includes multiple microphones and pressure transducers, signal amplifiers, analog-to-digital converters, time-code generators, and several laptops. While there are several lessons learned specific 
to the NASA AFRC equipment, the general lessons related to operator error, environmental considerations, and hardware considerations are of the greatest interest and are discussed below.

\section{Operator Error}

The ground instrumentation used by NASA AFRC consists of multiple ground microphones and pressure sensors distributed over large distances, which means that there are multiple operators of the recording equipment during a given test. Naturally, each operator is not equally experienced in using the equipment. A recurring problem has been the lack of data-acquisition-system operator training. Some data have been lost during several efforts because operators were not briefed or did not understand specific nuances of the equipment. While experienced users tend to be familiar with common problems and work-arounds for the systems they operate, new users are usually unaware that anything is wrong until the opportunity to collect the data has already passed. The lesson learned is that prior to the day of flight, new users must not only be briefed on how to use the system to which they are assigned, but they need the opportunity to practice using it. A checklist covering how to set up the equipment; highly detailed instructions covering how to operate the equipment; a list of common problems and idiosyncrasies with the equipment, as well as their solutions; and a short list of "final checks" for use immediately before the flight should be produced by experienced users and kept with the equipment. This method would mitigate the risk of a failure to record data during a flight, when the new operator would not have a chance to ask questions of someone with more experience. Using checklists for determining whether the equipment is ready to record greatly reduces the risk of leaving hardware powered-off during a test or otherwise failing to record the acoustic event.

Multiple times when data were collected incorrectly, it was done so for multiple flights. Having a method by which to quickly look at the data from each recorder and to confirm that the data were collected correctly could prevent incorrect data collection during multiple flights in a campaign. Looking at the data as soon as possible is helpful not only for the acoustic data, but for any type of recorded data. The lesson regarding the need to look at the data quickly between flights will be incorporated into future projects by building time into the flight schedule; however, developing a method to quickly determine data quality in the field would also be exceptionally useful.

\section{Environmental Considerations}

Lessons about the environment in which the sonic-boom team operates have been learned throughout the aforementioned sonic-boom flight research projects. Instrumentation cabling is very prone to being damaged by wildlife. Stowing outdoor cables overnight is an essential practice when instrumentation is in a place where wildlife has access to it. It was discovered that when operating on Rogers Dry Lakebed, keeping instrumentation cabling a distance of 500 feet or greater from the edge of the vegetation eliminated cable damage from wildlife. A commercial environmentally safe and biodegradable rabbit repellent was used to spray cables to attempt to reduce damage from wildlife, but had no effect. Coyotes also occasionally explore the equipment and, albeit rarely, defecate or urinate on equipment or storage boxes overnight. The lesson learned is that highly durable, watertight storage methods are crucial to protecting equipment from wildlife-related damage when the equipment must be stored outdoor overnight.

Easily-attached, removable dust covers for sensitive electronic connectors are also needed for connections that are left unplugged overnight. Other less-obvious environmental hazards include lawn sprinklers.

Sonic-boom teams have implemented methods to decrease the likelihood that environmental limits on instrumentation are exceeded. To do so, the team must understand how the instrumentation will behave in different weather conditions. On one occasion an automated sensor that was programmed to record sonic booms by way of a pre-set overpressure trigger experienced multiple false triggers due to the high winds of the day, in which gusts routinely exceeded the overpressure of the trigger. Midday heat also can lead to equipment shutting down or behaving incorrectly. Temperature swings can cause significant shifts in the calibration of instrumentation - including the frequency response of microphones. To mitigate these effects, reflective sun shields have been employed, and microphones are calibrated at the beginning and end of each day to account for the temperature swing.

\section{Hardware Considerations}

The configuration of the ground-recording equipment requires careful consideration. Due to the nature of sonic-boom testing, there is effectively only one chance to record the data (it is very costly to try to repeat a test when the data have not been recorded properly). Any personal computer (PC) being used to record data should have a minimum amount of software in addition to the data-recording software. Problems have occurred when an unrelated piece of software attempted to update during a critical time in the test. Security features, power-saving options, or other system settings that could cause problems at inopportune times should be turned off.

New equipment that has previously not been used should not be depended upon, and keeping the old equipment as backup is highly desirable. There have been close calls in the past when new equipment was delayed in the 
procurement process and did not arrive until the last minute, which was made worse by problems while using the new hardware. Having the old system to use just in case such problems arise can be critical. At least one backup unit should be available for each test-critical hardware component as a substitute in in the event of failure. Repair is usually not an option in the time available during the flight phase of the project, so it is important to have a plan of action should a piece of equipment fail. This plan of action could be anything from having a spare piece of equipment available for replacement, to adjusting the entire configuration of the equipment to compensate for a missing piece of equipment.

For large recording arrays containing many microphones, it is also important to have a plan for the deployment location of each piece of hardware and to label all hardware accordingly using gaffer tape. Appropriate cable management is also of the upmost importance, as it mitigates cable wear and failure.

Batteries have been the source of many problems during sonic-boom flight research projects; for instance, batteries have failed or prematurely drained during sonic-boom testing. Thus, it is extremely important to have multiple backups for batteries. Using equipment that operates on 12 volts direct current is very desirable, because motor vehicles can be used as a motor-generator when batteries fail. Having on hand the same number of battery chargers as batteries is also strongly recommended, because many batteries take multiple hours to charge, and having one charger per battery would enable charging all batteries overnight.

\section{B. Airborne Instrumentation}

In addition to the previously mentioned ground-based recording systems, NASA AFRC often utilizes an airborne instrumentation array. This array can include a TG-14 motorized glider (Aeromot, Brazil) and a small tethered blimp deployed from the back of a truck. Both are equipped with a small number of microphones; the data collected from these platforms serve to supplement the data collected on the ground.

\section{The TG-14 Motorized Glider}

The NASA AFRC uses a TG-14 motorized glider equipped with a wingtip-mounted microphone, called the Airborne Acoustic Measurement Platform, or AAMP, to record sonic booms at predetermined altitudes. The TG-14 is a two-place, side-by-side, motorized glider of a low wing, T-tail configuration, and is equipped with spoilers and retractable gear. A Garmin 496 global positioning system (GPS) (Garmin Ltd., Switzerland) with pre-programmed test waypoints assists in navigation. The TG-14 motorized glider is outfitted with a slightly modified version of the ground equipment.

Considerations concerning the performance of the TG-14 motorized glider require that the test team determine whether the TG-14 data are a requirement - attributed to the fact that the NASA AFRC F-18 airplane (McDonnell-Douglas, now The Boeing Company, Chicago, Illinois) is capable of a quick turnaround between passes, while the TG-14 motorized glider requires a long time to get back into position to begin the run-in for the next test point. This time is dictated not only by the TG-14 airspeed, but also because the TG-14 engine must remain off for the entire duration of the recording in order to decrease noise. The TG-14 motorized glider thus travels quite far from the run-in point and loses a significant amount of altitude over the duration of a single test point. The time lost can be reduced by setting the test waypoints to start at the highest altitude and progress to the lowest altitude and by avoiding large shifts in the coordinates of the run-in points. These optimizations reduce but do not eliminate the problem of the timing disparity between the F-18 airplane and the TG-14 motorized glider, so it is important to decide whether the F-18 airplane will proceed without the TG-14 motorized glider being in position. This decision needs to be communicated to the entire flight crew and addressed during crew briefs.

Another challenge related to the TG-14 airspeed became apparent during mission turnaround throughout the SCAMP. The remote location of the test site resulted in the TG-14 motorized glider spending a great deal of time flying from EAFB to the test site, which limited the number of flights that could occur in a day. If the ideal test site is remotely located, this location must be evaluated in light of the decreased data-gathering capability, and also weighed against the data-gathering requirements and desirements of the project.

A problem that has routinely resulted in data loss is operator error. The instrumentation is operated by the Flight Test Engineer while the pilot flies the test-point profile. The best results have been obtained when the operator was well-acquainted with the system, usually from extensive use of the equipment on the ground, as the equipment tends to require troubleshooting at least once per flight. A very good understanding of how the system works and a familiarity with common problems can speed up the troubleshooting process, providing a better chance of capturing all of the data.

Electromagnetic Interference - In the past, the TG-14 motorized glider caused electromagnetic interference (EMI) problems with the tablet PC, such that the propeller must be completely stopped before the tablet PC would function properly. If the tablet PC was powered on for any period of time while the TG-14 propeller was spinning, the tablet 
PC would stop operating, and require a reboot. It is suspected that this EMI problem was caused by the TG-14 magnetos, but the cause has yet to be confirmed due to time and workforce constraints. The lesson learned for future programs is to fully investigate the possibility of EMI with all instrumentation for all new configurations. The NASA AFRC updated the TG-14 instrumentation for future projects and completed thorough tests to ensure that no EMI problems manifested, resulting in a system that now requires very little troubleshooting during operation.

\section{Tethered Blimp}

The Airborne Acoustic Data Collection (AADC) system is owned and operated by Cessna Aircraft Company (Wichita, Kansas) and was used for the SCAMP and FaINT campaigns. The AADC is equipped with a radio-controlled flight termination system and can carry up to three Brüel \& Kjær (Nærum, Denmark) (B\&K) microphone recorders and GPS receivers.

The blimp has been used in Kansas with three B\&K model 2250 microphone data recording systems, and GPS data loggers. It was discovered during the SCAMP flight phase that the higher density altitudes of the high desert near NASA AFRC lowered the lifting capability, so only two microphone recorders could be carried. The lesson learned from tethered blimp operations is that equipment functionality can vary with location, especially when optimal operation relies on specific weather conditions. Equipment should be tested on-site prior to use and designed conservatively.

\section{Flight Phase}

Careful attention to detail during all previous phases of the sonic-boom flight research project increases the efficiency of the flight phase. During this phase it is important to revisit and emphasize each team member's duties. Clear and effective communication is crucial; the flight phase passes quickly but is consistently the busiest part of the project. For the purpose of this paper, the flight phase is defined as the time from when the fieldwork begins, until after the flights are complete and the supporting equipment is returned. Tasks to consider during the flight phase include test equipment deployment, operations, and retrieval; flight and field crew responsibilities; research team communications; and documentation.

\section{A. Test Equipment Deployment, Operations, and Retrieval}

Immediately prior to the flights, the workload of the project team begins to ramp up as the remaining coordination tasks are finalized and the team begins to prepare for the test. This section discusses the pre-test tasks that must be performed to ensure successful test execution and data analysis, including the pre-flight campaign, equipment deployment, and equipment pickup.

\section{Pre-flight Campaign}

The pre-flight campaign largely consists of preparing the test site and equipment for setup, and deployment of the equipment. This phase is relatively short, but begins a marked increase in workload for the team.

Surveying and Marking - Prior to the test, especially for cases in which knowing the exact placement of each microphone is critical to analysis, the position of each ground board and microphone should be surveyed and staked or marked. If the flags or marking will be removed when the instrumentation is installed or deployed, the survey data should be checked to ensure the accuracy and completeness of the survey prior to such removal.

Equipment Calibration - The acoustic recording equipment requires annual calibration. Some of the equipment can be calibrated by AFRC personnel, but some of the equipment must be sent to the manufacturer for calibration. When preparing for a test it is important that the project team be aware of the calibration processes and take into account the time required to complete these processes, especially when the manufacturer is involved. When arranging for the microphones to be calibrated, it is important to consider any tests expected to occur within the next year, because altering the configuration of the microphone invalidates the previous calibration.

The B\&K microphones can include a low-frequency adapter (LFA) or can be used without an LFA. Low-frequency adapters allow microphones to pick up lower frequencies, but unfortunately also attenuate the signal at higher frequencies. Therefore, if the shape of the sonic boom is more important than the noise-level data, then the LFA is acceptable or even preferable to use, depending on the frequencies being measured. If the data will be used to compute the perceived level of noise in decibels (PLdB) or played back for human testing, then LFAs should not be used. 


\section{Equipment Deployment}

Many of the NASA sonic-boom flight research projects involve deploying large microphone arrays in expansive secluded areas before the flight phase. The need to maximize the number of trained people available for equipment deployment cannot be overstated. The amount of time and physical effort required to deploy instrumentation is obviously directly proportional to the amount of workforce available. For reference, the FaINT project had adequate staffing: about 30 trained people deployed over 120 microphones.

Prior to deployment, all equipment and cables should be labeled and there should be a checklist indicating where each piece of equipment should be deployed. The project schedule should include dedicated time prior to deployment to check the contents of each pallet or container and perform a walkthrough of the deployment plan: what piece of equipment goes where, who is responsible for it, and in what order the equipment should be deployed.

Certain test-site locations present unique challenges. In publicly accessible areas there may be concern about people tampering with the equipment. Therefore, if equipment will be unattended for long periods of time in such areas, equipment housing should be rugged or a security detail should be employed. Rugged housings will also help prevent animals from damaging equipment. The most successful method of preventing unattended cables from being chewed is threading cable through flexible metal tubing; however, this method is time-intensive, and works best when there are only a few short cables. Animal repellents have been used, but with minimal or no success.

\section{Equipment Pickup}

The lessons learned for equipment teardown and pickup for NASA sonic-boom flight research projects are generally similar to those discussed for equipment deployment; however, there are additional distinct considerations. While most previous projects scheduled equipment pickup immediately after the last research flight, it is suggested that future project schedules include days after the flight phase completion for pickup. Such a schedule would result in shorter workdays as well as a less rushed process and more orderly stowing.

\section{B. Flight and Field Crew Responsibilities}

A significant amount of time prior to sonic-boom flight research testing should be spent considering what needs to be done each day and how to task team members accordingly. No single person or group should be overtasked, and each person needs to know their daily duties and how those duties fit into the big picture. Staffing in this manner limits chaos and presents a professional, united image to the project team (including external partners), any authoritative entities, and to any visitors to the test site.

Most importantly, the morning task load of the Principal Investigator (PI) needs to be managed. In the past, the PI has been responsible for calling in to the morning crew brief, performing the pre-flight planning, communicating the waypoints to the flight crew, shuttling people and equipment around the test site, and assisting with instrumentation setup and calibration. This workload is too much for one person to handle in the span of one to two hours, especially if there are problems to troubleshoot.

While the previous tests have been successful, the logistics of the tests would have been much less complicated if the project team had had more help from people who were consistently available and are well acquainted with the equipment and procedures of the sonic-boom flight research project team. Thus, it is recommended that a brief equipment familiarization training is provided to all field crew members internal to AFRC.

\section{Pre-flight Planning}

As mentioned above, the PI is responsible for daily pre-flight planning. Pre-flight planning is often performed using PCBoom, a code created and developed by Wyle Laboratories (El Segundo, California). To obtain flight waypoints for the pilots, the PI runs a MATLAB ${ }^{\circledR}$ (The MathWorks, Inc., Natick, Massachusetts) code that reads a weather and a trajectory file into PCBoom. This MATLAB ${ }^{\circledR}$ code is usually created by the PI. The PCBoom code will not run if the input file is not formatted correctly. Weather files sometimes contain a random error which is not easily visually apparent, so troubleshooting codes and inputs on the day of flight has occurred. Such troubleshooting, programming, or re-programming is not easy to accomplish when crew rest is lacking. Maintaining backup copies of codes and avoiding requirements creep in codes are both encouraged. For cases in which exposure levels over the duration of the sonic-boom flight research test are important, it is advisable to have separate, backup pre-flight planning code ready in case louder or quieter booms are needed.

\section{Research Team Communications}

One of the challenges unique to sonic-boom flight research projects is that it is essential for the field crew to communicate with each other and with the control room, but the field crew might be spread out over a large area. The solution is field radios, a communications hierarchy, and some creative thinking. 
The best tool for field communications and field-to-control room communications, based on past experience, is a set of LMRs. The control room can be set up to access the LMR frequency that is assigned to the test team, and the LMRs are very reliable, enabling timely communications during the flight.

During the sonic-boom flight research test it is critical that the field crew know when to expect the sonic boom so that any manually-triggered recording systems are activated at the correct time. The project team always briefs the predicted propagation time of the sonic boom during the crew brief or through electronic mail when the test points are sent to the flight crew. This practice helps the project team guess approximately when they should hear the sonic boom in the event that radios fail. If the radios are functional, the practice helps the field crew know how long the expected delay is between the "mark" call (indicating that the sonic-boom-generating airplane is at the proper flight conditions) and the sonic boom, so that no radio chatter occurs during this window, causing a loss or a corruption of data.

Hardware is important, but nothing more effectively facilitates communications during times of urgency than a well-considered protocol that is followed by all of the members of the project team. The NASA AFRC project team experienced the best outcome concerning communications protocol during the FaINT project. The FaINT project began with a Day One briefing, which included a discussion of the communications protocol, as well as a hands-on demonstration of the radios. The communications protocol should be created with two considerations in mind: safety and test efficiency. There is often a very short amount of time between passes during which the PI can relay trajectory changes to the pilots; doing so requires a quick survey of the qualitative field data (each field station reports what they heard) and making an assessment and decision based on that information.

Finally, participating in the pre- and post-flight crew briefs helps eliminate confusion between the pilots, the control room staff, and the field crew while providing context for any changes to operating procedures or communications protocols over the course of the test window. It is advantageous for the entire field team, or at least a few key leaders of the field team, to attend or call in to the pre- and post-flight crew briefs. Furthermore, field communications, procedures, and hardware operation being refined on a daily basis, a short all-hands meeting for the field crew at the beginning and end of each test day is a great time to communicate any new obstacles or concerns, while also allowing the project team to present their own concerns or obstacles. In order for these quick meetings to be useful, however, everyone needs to be present.

\section{Documentation}

Documentation is an important part of every flight phase. Thorough photography and field notes, including file logs, are critical to post-flight analysis. The tasks to ensure adequate documentation were learned over the course of the NASA AFRC sonic-boom flight research projects.

\section{Photography}

Extensive photography of the test setup is required for documentation. The resulting photograph set must include sensor locations and orientations in addition to detailing the placement of sensors relative to stationary landmarks. The relative placements can be utilized to relocate equipment if GPS survey data are not available. Prior to removing sensors, the photograph set should be checked to ensure that the required level of detail has been met.

\section{Field Notes}

Detailed, clear field notes are crucial during data processing and analysis. A field note template should be created and distributed to key field personnel prior to each flight. File logs, lists of calibration file names, test data, notes concerning extraneous noise, and times associated with test activity should be included in the field notes. If "stream of consciousness"-type comments are included, their meaning should be clarified, in writing on the same document, as soon as possible.

\section{Post-flight Phase}

The post-flight phase is easily overlooked, however the sonic-boom flight research project is not complete until the post-flight phase logistics have been completed, the project details have been recorded, the data have been processed, and appreciation has been communicated to supporting groups. To efficiently execute the post-flight phase tasks and set expectations with partner organizations the project team must also develop plans for the post-flight phase during project planning.

\section{A. Logistics}

The first item of business after test teardown is complete is to return borrowed equipment to the test partners. The effort required for this task is easy to underestimate. The sonic-boom flight research test should not be considered 
complete until all equipment has been returned to the owners. One person should be put in charge of this task, and should serve as the POC for communicating with shipping companies. Ideally, the POC is the equipment owner and the test is not concluded at the end of a work week, so that partner POCs are more likely to remain on site to supervise the return shipping process.

At the conclusion of a sonic-boom flight research test all involved parties will want to look at, or at least acquire, all of the data. While this desire is anticipated, accumulating and distributing the data is no simple task, and the expectations for receiving the data need to be managed. The timeline for gathering the data from the partners, wrapping all of the data up into one time-synced package, getting approval from export control to send the data out, and actually sending the data out needs to be discussed with the partners well in advance. It is preferable that this timeline is discussed prior to the test - during planning and coordination - on a general or rough-estimate level, while the more accurate and detailed timeline estimate should be presented to all parties during the Day One briefing.

\section{B. Recording Project Details}

The project details should be collected, evaluated, and recorded directly after the flight phase. The information compiled should include flight information, personnel involved, field notes, outputs, data files and formats, incidents (if any) and lessons learned. The documentation of these project details should be completed using an organized format with specific content required, such as a ground report. Recording these project details should be considered a priority and made readily available for future tests.

\section{Post-flight Data Processing}

Post-flight data processing should occur as soon as practical after the flight phase. Additionally, standard challenges to timely post-flight analysis should be recorded and addressed between projects to improve efficiency. For example, during the WSPR project, instances occurred in which the weather balloon terminated early, thus balloon information had to be pieced together from multiple balloons. A standardized technique for this task was created after the WSPR project to reduce future post-processing times.

\section{Communicating Appreciation}

Appreciation of supporting groups should be communicated very shortly after the conclusion of the sonic-boom flight research test. Including project accomplishments, in addition to how an organization specifically impacted the success, of the project helps build and maintain a great working rapport, thus aiding future tests.

As well, for tests with response volunteers, a formal and advertised "thank you" event can allow the team to simultaneously collect survey tools and distribute participation incentives.

\section{Conclusion}

While Sonic Booms on Big Structures (SonicBOBS), the Superboom Caustic Analysis and Measurement Project (SCAMP), Waveforms and Sonic boom Perception and Response (WSPR), and Farfield Investigation of No-boom Thresholds (FaINT) all were successful research efforts, a wealth of lessons learned resulted from each. Perhaps the biggest lesson learned is from consolidating all of these lessons into one document: it is critical to reflect on the planning and execution of a test before moving on to new endeavors and to document these lessons - otherwise these lessons might not be passed along to new team members or incorporated in future tests. The primary lesson to be gleaned from these tests is that it is of utmost importance to plan for tasks to take significantly longer than initially predicted to allow for buffer due to process, technical difficulty, troubleshooting, equipment malfunction, and other unknown unknowns. Secondly, always try to take load away from the test window by front-loading as much of the work as possible - including a thorough test-location checkout. Third, setting up the test schedule and tasking to allow data to be examined between flights can reduce lost data. Fourth, there is always a need for more crewmembers and prioritizing crew rest eliminates burnout and increases efficiency. Finally, the complexity of the test increases exponentially with the distance to any deployment.

\section{References}

[1] Haering, E. A. Jr., and Arnac, S. R., "Sonic Booms on Big Structures (SonicBOBS) Phase I Database,” DFRC-2020, March 2010.

[2] Page, J. A., et al., "SCAMP: Superboom Caustic Analysis and Measurement Project Overview," AIAA-2013-0930, January 2013.

doi: $10.2514 / 6.2013-930$

[3] Cliatt, L. J. II, et al., "A Flight Research Overview of WSPR, a Pilot Project for Sonic Boom Community Response," AFRC-E-DAA-TN15736, June 2014. 
[4] Cliatt, L. J. II, Hill, M. A., and Haering, E. A. Jr., "Mach Cutoff Analysis and Results from NASA's Farfield Investigation of No-boom Thresholds," AIAA-2016-3011, June 2016. doi: 10.2514/6.2016-3011

[5] Cliatt, L. J. II, Haering, E. A. Jr., Arnac, S. J., and Hill, M. A., "Lateral Cutoff Analysis and Results from NASA's Farfield Investigation of No-Boom Thresholds," NASA/TM-2016-218850, 2016.

[6] Haering, E. A. Jr. et al., "SCAMP: Rapid Focused Sonic Boom Waypoint Flight Planning Methods, Execution, and Results," AIAA-2013-0932, January 2013.

doi: $10.2514 / 6.2013-932$ 